\title{
Crecimiento vegetativo, producción y calidad de las fibras en plantas de algodonero sometidas a estrés mineral
}

\author{
Vegetative growth, yield and fiber quality of cotton plants \\ submmited to mineral stress \\ Fernando Broetto ${ }^{1 *}$, José Abramo Marchese $^{2}$, Renata Bruna dos Santos Coscolin ${ }^{1}$, \\ Érica Amanda de Barros ${ }^{1}$, Dayanne Fabrício Bressan ${ }^{1}$ e Marco Castillo Campohermoso ${ }^{3}$
}

\section{RESUMEN}

El potasio así como el nitrógeno constituyen los elementos necesarios para el crecimiento, desarrollo y producción del cultivo del algodón. Además, estos elementos están directamente relacionados con la formación de las fibras y cuando se proporcionan en forma adecuada, garantizan la calidad de las mismas. Con el objetivo de evaluar la influencia del potasio sobre parámetros biométricos, producción y calidad de la fibra del algodón, fueron cultivadas en invernadero plantas del cultivar Delpa opal, con fertilización potásica alterada, simulando deficiencia (subdosis) y dos niveles de sobredosis. Durante las diferentes fases fenológicas del cultivo fueron analizadas variables biométricas incluyendo la producción de fibras y sus parámetros cualitativos. Los tratamientos de subdosis de potasio y las sobredosis aplicadas alteraron todos los parámetros evaluados, demostrando la importancia de la fertilización potásica sobre los mismos.

Palabras clave: Biometría, estrés, potasio, calidad de fibra.

\begin{abstract}
Potassium, like nitrogen, it constitutes an essential element for growth, development and production of cotton crops. Moreover, these elements are directly related to fiber formation and when provided in suitable doses, influences its quality. The aim of the experiments was to evaluate the influence of potassium on biometric parameters, yield and quality of cotton fibers. For this purpose, plants of the cultivar Delta opal were grown in a greenhouse with altered potassium fertilization, simulating $K$ deficiency and two overdoses. During the different phenological stages, biometric variables were analyzed including the production of fibers and their qualitative parameters. The sub-dose and the overdoses treatments of $K$, changed all the evaluated parameters, demonstrating the importance of potassium fertilizer on them.
\end{abstract}

Key words: Biometrics, fiber quality, potassium, stress.

\section{Introducción}

El algodón es una planta de clima tropical, aunque algunos cultivares se desarrollan en regiones de leve temperatura o en regiones de temperatura semiárida. El cultivo requiere de la humedad del suelo para la germinación de la semilla a partir del período de desarrollo de la plántula y especialmente en el período comprendido entre la formación de los botones florales y el inicio de la aparición de los frutos (35 a 120 días del ciclo de vida). En términos nutricionales se destacan los estudios sobre la importancia para el desarrollo de la planta y la producción de fibras. El potasio (K) está asociado al transporte del producto de la fotosíntesis y la producción de almidón. Su presencia es fundamental en el rendimiento de las raíces y en el contenido de materia seca total, siendo considerado un nutriente requerido en grandes cantidades por las plantas (Dechen et al., 1999). Es un elemento móvil y su deficiencia se observa en las hojas más viejas, ya que se trasloca a las hojas más nuevas, siendo su nivel crítico foliar de $1,2 \%$ y en los pecíolos de 2,5\% (Ting, 1982).

Según Ting (1982), la deficiencia de potasio se puede notar por los siguientes síntomas: ramificación

\footnotetext{
Instituto de Biociências, UNESP, Botucatu, SP 18618-000, Brasil.

Lab. de Bioquímica e Fisiología Vegetal, Faculdade de Agronomía, UTFPR, Pato Branco, PR 85503-390, Brasil.

Centro de Edafología y Biología Aplicada del Segura (CSIC), Dep. de Riego-P.O. Box 164, E-30100 Murcia, España.

*Autor correspondiente: broetto@ibb.unesp.br
}

Fecha de Recepción: 02 Abril, 2012.

Fecha de Aceptación: 25 Septiembre, 2012. 
excesiva, mucho follaje y pocas raíces; entrenudos cortos, pecíolos cortos, hojas pequeñas, amarillamiento y necrosis en el borde de las hojas.

Rosolem (2001) sitúa al potasio como componente importante en el aumento de la productividad y la mejora de la calidad de la fibra del algodón. Además, este nutriente está relacionado con la resistencia de las plantas contra plagas y enfermedades. La nutrición potásica adecuada contribuye para que las fibras del fruto se estiren, permanezcan más finas y resistentes (Grespan \& Zancanaro, 1999). Además, el potasio regula el ritmo de crecimiento del algodón, favoreciendo el aumento de la materia seca dos capullos, lo que se refleja en una mayor productividad, madurez y micronaire de las fibras (Silva, 2001). Estudios realizados por Read et al. (2006) indican que el K y el N están involucrados directamente con la producción y calidad de la fibra. Especialmente los autores observaron que la deficiencia/estrés del nitrógeno $(\mathrm{N})$ afecta el crecimiento y longitud de la fibra, mientras que la deficiencia de $\mathrm{K}$ parece estar más relacionada con aspectos reproductivos y movimiento de azúcares en las plantas del algodonero. De acuerdo con Fortuna et al. (2001), el aumento en el suministro de $\mathrm{N}$ y K puede modificar algunas características de la fibra del algodón, como resistencia y alargamiento. Niveles excesivos de $\mathrm{N}$ y K aumentan la masa de los capullos además de otras características cualitativas de las fibras, por otro lado podemos provocar también una disminución de la resistencia, estiramiento y aumento en el número de neps (índice de irregularidades de hebras) por gramo (Lauchli et al., 1981). De acuerdo con estos autores el exceso de $\mathrm{Na} \mathrm{y} \mathrm{Cl}$ en la planta (como productos de la disociación de $\mathrm{NaCl}$ y $\mathrm{KCl}$ ) en el protoplasma podemos generar efectos negativos en el balance iónico $\left[\mathrm{K}^{+}\right.$y $\mathrm{Ca}^{+}$, con relación al $\mathrm{Na}^{+} ; \mathrm{Cl}^{-}$en relación al (SO4) ${ }_{2}^{-}$, induciendo efectos secundarios en los iones sobre la actividad enzimática y actividades celulares. La época de plantación tardía, combinada con la deficiencia de $\mathrm{K}$, presentó un efecto sinérgico sobre la producción y calidad de las fibras del algodón (Gormus \& Yucel, 2002). En este trabajo, los autores demostraron que la aplicación de $\mathrm{K}$ en el suelo (150 kg K $\left.\mathrm{O} \mathrm{ha}^{-1}\right)$ disminuyó el número de días requeridos para que las plantas emitiesen los primeros capullos, comparando las plantas cultivadas en ausencia de $\mathrm{K}$ (control).

La deficiencia de $\mathrm{N}$ y $\mathrm{K}$ interfiere en la formación de los frutos del algodonero, principalmente en la parte superior de la planta, alterando la calidad de las fibras (Fortuna et al., 2001). Trabajando en ambiente protegido aumentando la concentración de $\mathrm{CO}_{2}$, Reddy \& Zhao (2005) demostraron que la deficiencia de $\mathrm{K}$ suprimió el crecimiento y otros factores fisiológicos del algodonero por el enriquecimiento de la atmósfera $\left[\mathrm{CO}_{2}\right]$. De esta manera, es posible que las alteraciones ambientales debido al aumento de las concentraciones de $\mathrm{CO}_{2}$, deberán ser acompañadas de un consumo cada vez mayor de nutrientes en especial el K para garantizar la productividad. El presente ensayo tuvo como objetivo estudiar los efectos fisiológicos de la deficiencia o exceso de potasio aplicado a las plantas de algodonero, en ambiente protegido. Como complemento del experimento se evaluaron parámetros biométricos y producción de fibras, así como sus aspectos cualitativos.

\section{Material y Métodos}

\section{Material vegetal, condiciones de crecimiento y tratamientos}

El experimento fue realizado en condiciones de invernadero, en el Departamento de Ingeniería Rural de la Facultad de Ciencias AgronómicasUNESP-Botucatu, SP-Brasil.

Las semillas de algodonero (Gossypium hirsutum L.) cv. Delta Opal fueron sembradas en macetas con capacidad de 20 litros. Antes de plantarlas, el suelo utilizado en el experimento fue colectado en la granja experimental Lageado, Série Patrulha, fue tamizado y neutralizado a través de encalado y suplementado con fertilización básica. Antes del encalado y la fertilización de base, las muestras de suelo fueron analizadas en el departamento de Producción Vegetal de FCA/UNESP-Sector de Suelos y Nutrición Mineral. Basado en el análisis se calculó la cantidad de calcio dolomítico necesario para elevarlo V (saturación de bases) hasta 70\%. El suelo después del encalado, se humedeció y se sometió a incubación por 45 días. Para los cálculos de las necesidades de calcio (NC), se utilizó la siguiente expresión:

$\mathrm{NC}=\mathrm{CTC} \times(\mathrm{V} 2-\mathrm{V} 1) / 10 \mathrm{x}$ PRNT; donde $\mathrm{NC}=$ necesidad de calcio, $\mathrm{CTC}=$ capacidad de intercambio catiónico, $\mathrm{V} 1=$ bases intercambiables (\%) en el suelo; V2= bases intercambiables (\%) requeridas para las plantas; $\mathrm{PRNT}=$ poder relativo de neutralización total. 
La fertilización de base fue realizada de acuerdo con lo recomendado por el boletín 100 de IAC (Instituto Agronómico de Campinas. Campinas-SP, Brasil), para el cultivo de algodonero. El fósforo fue aplicado en dosis de $150 \mathrm{ppm}$ - SST; el nitrógeno en forma de $\mathrm{NHSO}_{4}(50 \mathrm{ppm})$ y como fuentes de micronutrientes, se aplicaron $\mathrm{B}\left(2 \mathrm{ppm}-\mathrm{H}_{3} \mathrm{BO}_{3}\right)$ y $\mathrm{Zn}\left(2 \mathrm{ppm} \mathrm{ZnSO}_{4}\right)$. El potasio, por tratarse de un macronutriente y ser estudiado como factor estresante, fue aplicado en solución, cuando las plantas tenían 60 días después de la siembra (DPS), coincidiendo con los primordios de la fase de floración. $\mathrm{El} \mathrm{KCl}$ fue aplicado en forma parcelada (2 veces) con intervalo de 7 días entre aplicaciones. Al tratamiento control (C) se aplicaron $150 \mathrm{ppm}$ de $\mathrm{KCl}$ (dosis recomendada por Raij et al., 1996). Los demás tratamientos fueron una subdosis (SD) con 75 ppm de $\mathrm{KCl}$ y dos superdosis (S1 y S2), con 225 y $300 \mathrm{ppm}$ de $\mathrm{KCl}$, respectivamente. De esta manera los tratamientos se denominaron como $\mathrm{C}=$ control; $\mathrm{SD}=$ subdosis; $\mathrm{S} 1=$ superdosis $1 ; \mathrm{S} 2=$ superdosis 2 .

Paralelamente se aplicó una dosis de N (80 ppm) en dos parcelas, complementaria a la fertilización de base.

El diseño experimental fue en bloques al azar (C, SD, S1 y S2), con 15 macetas para cada tratamiento, dando un total de 60 macetas. Las plantas fueron regadas diariamente, el suelo se mantuvo con buena disponibilidad de agua. Las plantas fueron evaluadas con respecto a sus características fenológicas en los diferentes estadios de crecimiento con las determinaciones de altura de plantas y número de capullos/flores.

Las medidas de altura de plantas así como el número promedio de flores y capullos fueron realizadas a los $15,30,45,60$ y 75 días después de la aplicación de los tratamientos. El número de plantas evaluadas fue con una media de 28 plantas por tratamiento $(n=2)$, considerándose que cada maceta se mantuvo con dos plantas.

\section{Área foliar}

El área foliar de 20 hojas para cada uno de los cuatro tratamientos fue analizada en un escáner area meter mod. LICOR LI-3000. El análisis fue realizado a los 75 días después de la aplicación de los tratamientos, coincidiendo con la fase de maduración de los capullos. Para su determinación, las hojas fueron colectadas cuando estaban completamente expandidas en la región media de las plantas (posición 5 a partir del ápice), se metieron en bolsas de plástico y se midieron en un máximo de 3 minutos después de la colecta.

\section{Determinación de la masa (MF) de los componentes de producción}

Los capullos fueron colectados cuando estaban totalmente desarrollados, abiertos y sin presencia de tejidos verdes $(n=15)$. El material se separó en tres partes: fibra, semillas y capullos, se determinó por peso la masa de cada componente en g MF planta ${ }^{-1}$.

\section{Evaluación de las características agronómicas de la fibra}

Para cada uno de los bloques de los tratamientos fueron colectadas muestras aleatorias de $10 \mathrm{capu}-$ llos, en la región tercio media de las plantas, para los análisis de la fibra. El análisis fue procesado por el laboratorio de Tecnología de Fibras de IAC/ APTA en Campinas, SP, con un equipo HVI 1000 M700/USTER.

\section{Análisis estadístico}

Se realizó un diseño completamente al azar. Los resultados obtenidos en las evaluaciones fueron sometidos a un análisis de varianza por la prueba de Tukey con un nivel de 5\% de significancia.

\section{Resultados y Discusión}

\section{Evaluaciones biométricas}

Para investigar el efecto de diferentes concentraciones de $\mathrm{KCl}$ sobre el crecimiento de las plantas de algodonero, se evaluó la altura de las plantas, medida hasta la base de la yema apical. Antes de la aplicación de los tratamientos (a los 60 DPS), se hizo raleo manteniéndose dos plantas por maceta para cada tratamiento, se hicieron 28 determinaciones por tratamiento. Se observó (Figura 1) que el tratamiento control presentó tasas de crecimiento lineales, estabilizándose en la fase de desarrollo de los capullos (colecta 5, T5) a los 75 días después de la aplicación de los tratamientos. Se observó que los tratamientos SD (subdosis) y las dos superdosis utilizadas (S1 y S2) limitaron el crecimiento de las plantas, cuando se compararon con el control. 


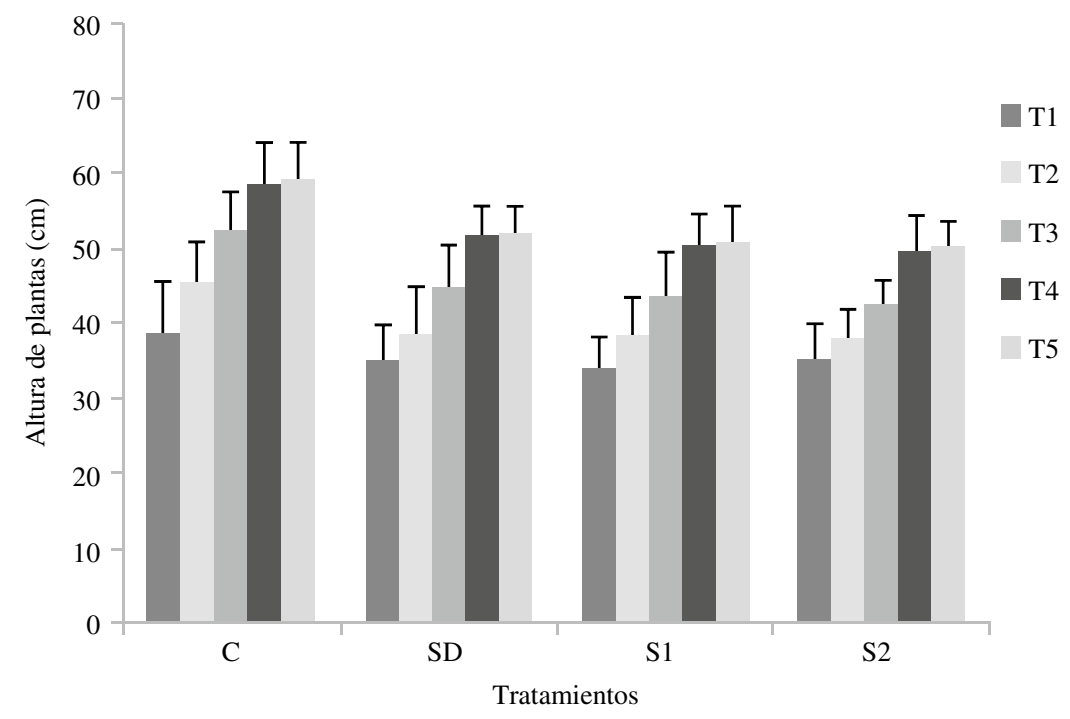

Figura 1. Altura de las plantas de algodonero cultivadas bajo diferentes concentraciones de $\mathrm{KCl}$ y medidas a los $15,30,45,60 \mathrm{y}$ 75 días después del inicio de los tratamientos (T1 a T5). Los tratamientos representan el control (C), Subdosis (SD), Superdosis 1 (SD1) y Superdosis 2 (SD2). Los valores representan una media \pm SE $(n=28)$.

Aunque el algodonero puede presentar cierta tolerancia a la salinidad, el exceso de sal puede reducir el crecimiento de las plantas (François, 1982). Es posible que el exceso de $\mathrm{KCl}$ haya inducido alto índice salino en el tejido vegetal. Este efecto probablemente interfiere en el metabolismo general de las plantas alterando patrones de síntesis proteica, actividad enzimática, etc. Las plantas generalmente presentan tasas de crecimiento reducidas como estrategia para direccionar energía metabólica y así tolerar los efectos del estrés salino. Estas estrategias están muchas veces relacionadas con la disminución de tasas fotosintéticas y al cierre estomático (Munns \& Tester, 2008), permeabilidad de las membranas, actividad de canales de agua (acuaporinas) y balance iónico (Álvarez et al., 2009), los cuales son factores limitantes de crecimiento.

Se observó también que a concentraciones altas de $\mathrm{KCl}$ (tratamiento $\mathrm{S} 2$ ), la disminución de la tasa de crecimiento fue discreta, en el cv. Delta Opal mostrando relativa tolerancia al estrés mineral. De igual manera la subdosis utilizada (tratamiento SD) parece no ser suficiente para alterar significativamente el parámetro de crecimiento. Aparentemente, el algodonero puede utilizar mecanismos moleculares para resistir condiciones adversas.

El área foliar (Figura 2) fue reducida en los tres tratamientos de $\mathrm{KCl}$, cuando se compararon con el control independientemente del nivel aplicado (SD,
S1 y S2). Sin embargo, no se observaron diferencias estadísticas entre ellas. La disminución del área foliar puede estar relacionada con la estrategia de reducir las pérdidas de agua vía transpiración y contribuir a la economía del agua de las plantas

Como ya se mencionó anteriormente, se observó también una tendencia a disminuir la altura de las plantas, de la misma manera que se observó para el área foliar, o sea, en función de los tratamientos con $\mathrm{KCl}$. Estos resultados pueden indicar estrategias adoptadas por las plantas, basados en la economía del agua, este parámetro puede estar relacionado con una disminución en la productividad. La menor área foliar contribuyó para el proceso de adaptación de las plantas bajo estrés causado por el efecto osmótico del fertilizante en el suelo, concordante con Mateo (1999). Como síntoma inicial, el estrés de la sal de $\mathrm{KCl}$ induce una restricción de las plantas a la expansión foliar (Alarcón et al., 1993), ya que una reducción del área foliar está íntimamente relacionada con mecanismos de prevención, los cuales pueden minimizar las pérdidas de agua, principalmente debido al cierre estomático.

Otro parámetro de crecimiento evaluado fue el número medio de flores y capullos producidos por las plantas durante la fase juvenil a la fase adulta. Debido a que los tratamientos fueron aplicados al inicio de la floración la evaluación consideró número de flores y capullos formados (Figura 3). 


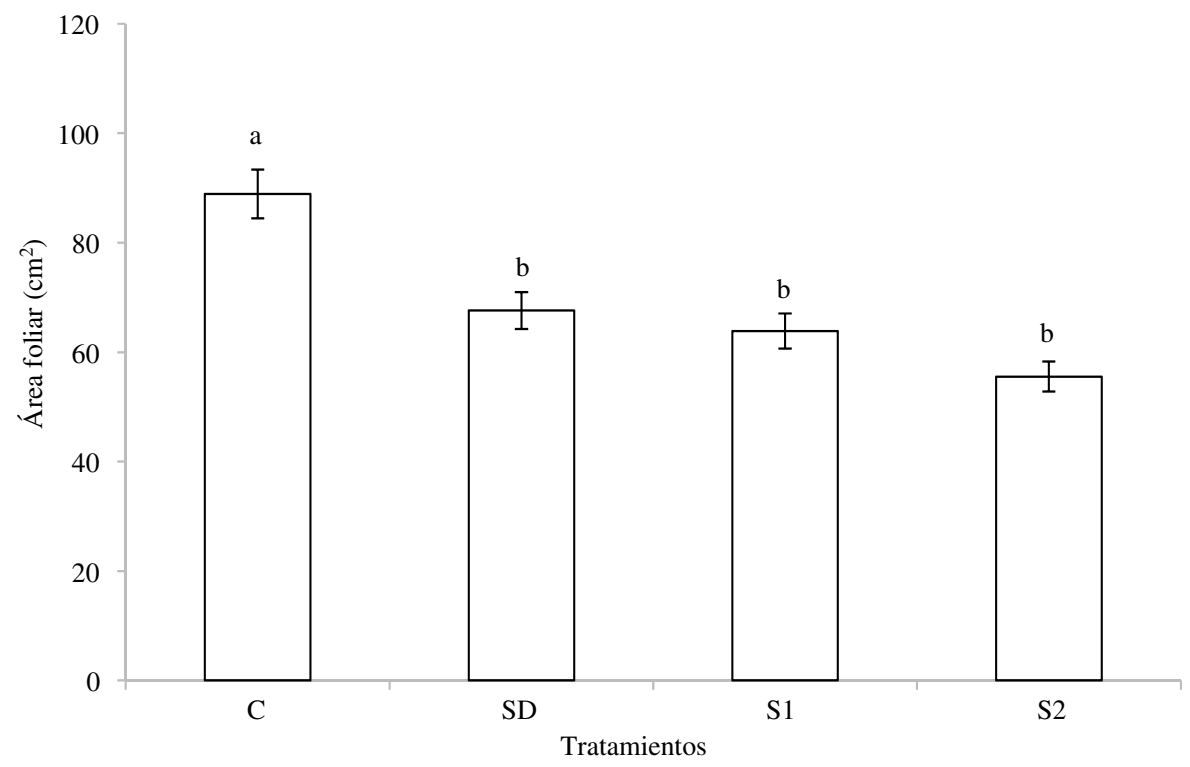

Figura 2. Área foliar en plantas de algodonero sometidas a estrés mineral. El análisis fue realizado a los 75 días después de la aplicación de los tratamientos, coincidiendo con la fase de maduración de capullos. Los valores representan una media $\pm \mathrm{SE}(\mathrm{n}=28$ ), comparadas por la prueba de Tukey e identificadas por letras $(\mathrm{P}<0.05)$; Letras iguales, no representan diferencias significativas.

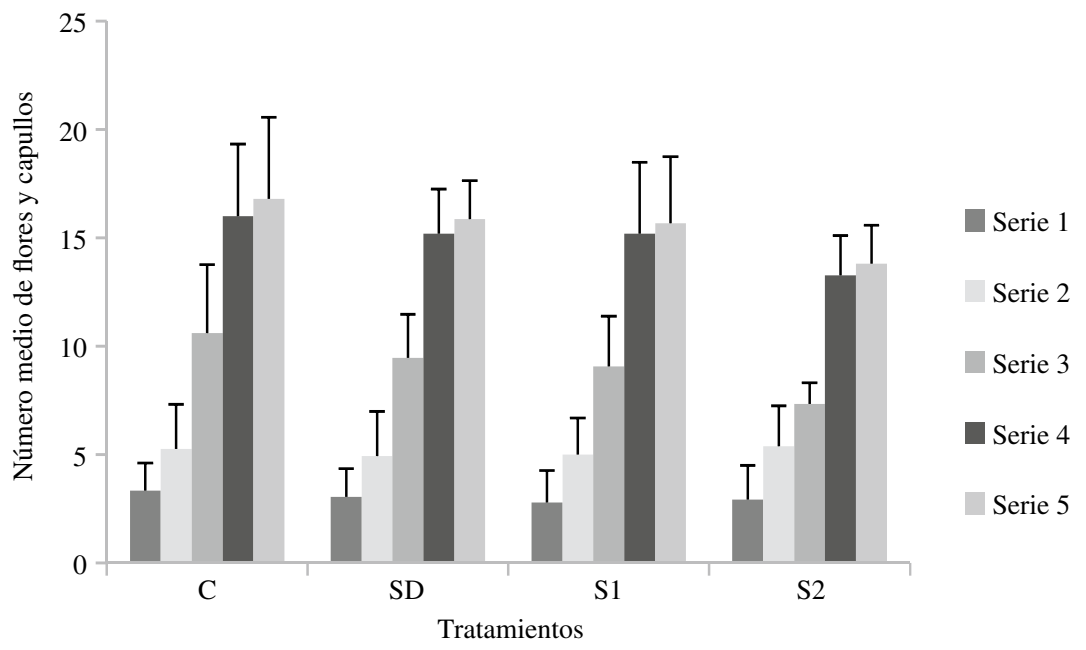

Figura 3. Número medio \pm SE de flores y capullos formados por planta $(n=15)$ en plantas de algodonero cultivadas bajo diferentes concentraciones de $\mathrm{KCl}$. Las medidas fueron efectuadas a los 15, 30, 45, 60 y 75 días después del inicio de los tratamientos (T1 a T5). los tratamientos representan el control (C), Subdosis (SD), Superdosis 1 (S1) y Superdosis 2 (S2).

Como se puede observar en la Figura 3 el tiempo de muestreo 3 (T3) a los 45 días después de la aplicación de los tratamientos parece ser el período en que la formación de los capullos fue más afectada. Por otra parte, en los últimos muestreos este efecto parece haber sido controlado, con poca variación negativa de los tratamientos en relación con el control. Esto no significa que la producción de fibra y calidad de la misma pueda presentar un mismo resultado, considerándose que las cinco evaluaciones presentaron diferencias en relación con el control.

El crecimiento de las plantas de algodonero (altura) parece ser por el aumento de la CE (conductividad eléctrica) de la solución del suelo. La conductividad eléctrica en la solución del suelo a $4 \mathrm{dS} \mathrm{m}^{-1}$ puede estimular el crecimiento, siendo este valor considerado próximo al límite, a partir del cual las plantas pueden inhibir su crecimiento, 
aunque Maas \& Hoffman (1977) consideren que este valor puede alcanzar 7,7 $\mathrm{dS} \mathrm{m}^{-1}$ sin interferir el crecimiento de la especie.

El aumento del índice salino en la solución del suelo perjudica la absorción del agua y otros elementos minerales, pudiendo así reducir la productividad fotosintética y síntesis de asimilados. Obviamente, este efecto culmina con un bajo rendimiento del cultivo. De acuerdo con estas citas, en el presente ensayo las plantas fueron sometidas a CE bajo estos límites de tolerancia (E2 alcanzó una media de $2,8 \mathrm{dS} \mathrm{m}^{-1}$ ), lo que de cierta forma justifica la respuesta observada.

\section{Componentes de la producción}

Los capullos fueron colectados cuando estaban totalmente desarrollados, abiertos y sin presencia de tejidos verdes. Se separó el material en tres partes: fibra, semillas y capullos, se determinó la masa en g planta $^{-1}$, cuyas masas están representadas en la tabla 1 .

Los tratamientos con mayor aplicación de $\mathrm{KCl}$ (S1 y S2) claramente interfirieron en la producción de las fibras. Se observó una reducción en la producción de la fibra comparada con el control (celulosa pura) alcanzando $18,9 \%$ para S1 y $27,6 \%$ para la superdosis
2 (S2) de $\mathrm{KCl}$. En relación con la subdosis utilizada (SD), la disminución en la producción fue alrededor del $12 \%$. Por lo tanto, el exceso de $\mathrm{KCl}$ se refleja en la producción del algodón; esto lo confirman algunos datos relativos al número de capullos y altura de las plantas evaluados en el experimento.

La materia fresca de las semillas también disminuyó en función de los tratamientos, pero en menor intensidad. El tratamiento S1 y S2 no presentan diferencias entre sí, además $\mathrm{S} 1$ tampoco fue diferente al tratamiento SD. El control fue superior a todos los demás tratamientos. $\mathrm{La}$ materia fresca de capullos presentó un resultado estadístico semejante entre los tratamientos con exceso de $\mathrm{KCl}$, diferentes al SD y al tratamiento control. También se observó que los tratamientos que tenían alguna forma de estrés fueron afectados cuando se compararon con el control.

Como es conocido para varias especies vegetales, la salinización del suelo puede inducir, por un lado, efectos osmóticos que limitan la absorción del agua y, por el otro, efectos iónicos, los cuales debido a la acumulación de sodio, potasio y cloro son tóxicos para las células (Cassaniti et al., 2009). Ambos procesos pueden suprimir las tasas de crecimiento y otros factores metabólicos, variando de

Tabla 1. Producción de algodón en función del tratamiento con diferentes dosis de KCl. Los valores indican una media (n=15) de la materia fresca (MF) de capullos completamente secos, colectados durante todo el experimento hasta el final del ciclo del cultivo.

\begin{tabular}{cccc}
\hline Tratamientos & MF de la fibra & MF de semillas & MF de capullos \\
\hline Niveles de KCl & g planta $^{-1}$ & g planta $^{-1}$ & g planta $^{-1}$ \\
C = Control & $11.63 \mathrm{c}$ & $14.03 \mathrm{c}$ & $13.87 \mathrm{c}$ \\
SD $=$ Subdosis & $10.21 \mathrm{~b}$ & $12.41 \mathrm{~b}$ & $11.60 \mathrm{~b}$ \\
S1 = Superdosis 1 & $9.43 \mathrm{~b}$ & $11.71 \mathrm{ab}$ & $10.69 \mathrm{a}$ \\
S2 $=$ Superdosis 2 & $8.42 \mathrm{a}$ & $10.87 \mathrm{a}$ & $10.60 \mathrm{a}$ \\
\hline
\end{tabular}

Letras iguales no representan diferencias significativas a un nivel de $5 \%$ por la prueba de Tukey $(\mathrm{P}<0.05)$.

Tabla 2. Valores medios $(n=10)$ de las características agronómicas de la fibra de algodón en función del tratamiento con diferentes dosis de $\mathrm{KCl}$. Medias comparadas por la prueba de Tukey, con una significancia de $(\mathrm{P}<0.05)$.

\begin{tabular}{ccccccccccc}
\hline Tratam. & UHML & UNIF & SFI & STR & AL & MIC & MAT & REFL & COR & SCI \\
\hline C & $25.8 \mathrm{a}$ & $83.7 \mathrm{~b}$ & $6.2 \mathrm{~d}$ & $28.1 \mathrm{c}$ & $6.6 \mathrm{a}$ & $4.3 \mathrm{a}$ & $86.0 \mathrm{a}$ & $82.7 \mathrm{c}$ & $10.1 \mathrm{a}$ & $131 \mathrm{~d}$ \\
S1 & $26.1 \mathrm{~b}$ & $83.7 \mathrm{~b}$ & $4.6 \mathrm{a}$ & $27.8 \mathrm{~b}$ & $7.1 \mathrm{~b}$ & $4.7 \mathrm{~b}$ & $86.0 \mathrm{a}$ & $80.1 \mathrm{~b}$ & $10.7 \mathrm{~b}$ & $126 \mathrm{c}$ \\
S2 & $26.6 \mathrm{c}$ & $82.7 \mathrm{a}$ & $5.8 \mathrm{c}$ & $28.1 \mathrm{c}$ & $6.6 \mathrm{a}$ & $5.0 \mathrm{c}$ & $88.0 \mathrm{a}$ & $79.1 \mathrm{a}$ & $10.3 \mathrm{a}$ & $119 \mathrm{a}$ \\
SD & $26.6 \mathrm{~b}$ & $83.3 \mathrm{~b}$ & $5.2 \mathrm{~b}$ & $27.1 \mathrm{a}$ & $7.2 \mathrm{~b}$ & $4.7 \mathrm{~b}$ & $86.0 \mathrm{a}$ & $80.0 \mathrm{~b}$ & $10.8 \mathrm{~b}$ & $123 \mathrm{~b}$ \\
\hline
\end{tabular}

$\mathrm{UHML}=$ Longitud media de la mitad superior $(50 \%)$ de las fibras de la muestra $(\mathrm{mm}) ; \mathrm{UNIF}=$ Índice de Uniformidad de longitud (en \%); SFI= Índice de fibras cortas (en \%); STR= Tenacidad de ruptura de la fibra (en g/tex); AL= extensión de ruptura de la fibra (en \%); MIC= Micronaire de la fibra; MAT= Índice de madurez de la fibra, en \%; REFL= Reflectancia de la fibra, Rd \%; COR= Índice de amarillamiento de la fibra, $+\mathrm{b}$; SCI= Previsión de la fiabilidad. 
intensidad de acuerdo con la concentración salina y la capacidad de tolerancia de la especie.

Altas concentraciones de sal debido a la aplicación de $\mathrm{KCl}$ pueden producir efectos iónicos expresivos, los cuales pueden tener efecto principalmente en la síntesis proteica con aumento en la actividad de enzimas antioxidativas. El límite de tolerancia de las plantas depende de la concentración de la sal en la solución, tiempo de exposición así como el estado de desarrollo de las plantas (Ayers \& Westcot, 1991).

A pesar de la existencia de variabilidad genética para la tolerancia a la salinidad (Shannon \& Grieve, 1998), los mecanismos bioquímicos y fisiológicos que contribuyen para dicha tolerancia todavía son poco conocidos. Aplicaciones de dosis elevadas y continuas de $\mathrm{KCl}$ en el suelo pueden también elevar el contenido de cloro en la planta, provocando una clorosis y necrosis de las hojas, además de una reducción en la producción. De acuerdo con Malavolta et al. (1997), el cloro no entra en la formación de compuestos orgánicos, siendo necesario para la fotolisis del agua, durante la fotosíntesis y transporte eléctrico.

\section{Evaluación de las características agronómicas de la fibra}

Los indicadores mundiales de calidad de fibra del algodón indican que el comportamiento medio varía entre 26,5 y 31,2 mm, en algunas regiones de Brasil (Bahia y Mato Grosso), estos valores pueden alcanzar medias de 28,9 mm (Fonseca, 2006). Se observó que en los tratamientos del presente experimento presentaron valores de comportamiento de fibra (UHML) dentro del rango esperado, excepto el control, que presentó un valor ligeramente menor. Aparentemente, valores más elevados de $\mathrm{K}$ (tratamiento S2) y la subdosis expresaron mejor resultado para este parámetro (tabla 2).

En relación con el índice de micronaire de la fibra (MIC), los valores de referencia para Brasil están en el rango de 3,8 a 4,2. De la misma forma que lo observado para la longitud, también los índices de MIC en los tratamientos de estrés (S1 y S2) fueron superiores al control, con diferencias estadísticas por la prueba de Tukey. Este resultado concuerda con los obtenidos por Sabino et al. (1995). Estos autores observaron índices de MIC en proporción a la aplicación de $\mathrm{K}$ en la forma de $\mathrm{K}_{2} 0$ entre 0 y $150 \mathrm{~kg} \mathrm{ha}^{-1}$.

Otros parámetros estudiados, como la uniformidad en la longitud (UNIF\%) e índice de madurez de la fibra (MAT) revelaron baja interferencia en los tratamientos cuando se compararon con el control.

Sabino et al. (1995) estudiaron el efecto del potasio sobre las características tecnológicas de la fibra del algodonero. En este trabajo los autores discuten que la deficiencia del elemento mejoró parámetros tecnológicos de la fibra como la uniformidad de longitud, índice de micronaire y madurez. Sabino et al. (1995) concluyeron que la fertilización potásica elevó la masa de los capullos y de semillas, así como la uniformidad de longitud e índice de micronaire. La aplicación de potasio también mejoró la tenacidad, lo que refleja la mejor resistencia promedio por fibra individual.

En condiciones de deficiencia de potasio se pueden esperar efectos agronómicos sobre la calidad de la fibra, como uniformidad de longitud, longitud y micronaire (Pettigrew \& Meredith, 1997).

\section{Conclusiones}

Las dosis de $\mathrm{KCl}$ aplicadas a las plantas de algodonero alteran notoriamente parámetros biométricos, interfiriendo en la producción y calidad de las fibras, el efecto más marcado fue debido a la subdosis y a la mayor concentración de potasio.

\section{Agradecimientos}

Este trabajo fue financiado por la FAPESP, a través del contrato 04270-1 concedido a F.B.

\section{Referencias Bibliográficas}

Alarcón, J.J.; Sánchez-Blanco, M.J.; Bolarín, M.C.; Torrecillas, A. 1993 Water relations and osmotic adjustment in Lycopersicon esculentum and L. Pennellii during short-term salt exposure and recovery. Physiologia Plantarum, 89: 441-447.

Álvarez, S.; Gómez-Bellot, M.J.; Bañón, S.; Sánchez-Blanco, M.J. 2009 Respuesta fisiológica y agronómica de plantas de Phlomis purpurea con baja disponibilidad hídrica y regadas con agua salina. In: XVIII Reunión de la Sociedad Española de fisiología Vegetal y XI Congreso Hispano-Luso de Fisiología Vegetal. Zaragoza España. p. 410.

Ayers, R.S.; Westcot, D.W.

1991 A qualidade da água na agricultura. Campina Grande: UFPB, 218 p. (Estudos FAO. Irrigação e drenagem, 29). 
Cassaniti, C.; Leonardi, C.H.; Flowers, J.T.

2009 The effects of sodium chloride on ornamental shrubs. Scientia Horticulturae, 122: 586-593.

Dechen, A.R.; Furlani, A.M.C.; Furlani, P.R. Tolerância e adaptação de plantas aos estresses nutricionais.

1999 In: SIQUEIRA, J.O. Interrelação fertilidade, biologia do solo e nutrição de plantas. Viçosa: Sociedade Brasileira de C.S. Lavras: Universidade Federal de Lavras/ Departamento de Ciências do Solo, pp. 337-362.

Fonseca, R.G.

2006 Qualidade global da fibra de algodão produzida no cerrado brasileiro. Embrapa, Circular Técnica, Brasília, 7 p.

Fortuna, P.A.; Raimundo, J.; Balada, W.R.

2001 "Produtividade e qualidade de fibra do algodão (Gossypium hirsutum L.) em função de doses de N e K na Fazenda Sucuri-Grupo Sachetti-safra 00/01.' In: III Congresso Brasileiro de Algodão, 2001, Campo Grande (MS). Anais do III Congresso Brasileiro de Algodão. Dourados (MS) : UFMS e Embrapa Agropecuária Oeste, pp. 1064-1066.

François, L.E.

1982 Narrow row cotton. (Gossypium hirsutum L.) under saline conditions, Irrg. Sci., 3: 149-156.

Gormus, O.; Yucel, C.

2002 Different planting date and potassium fertility effects on cotton yield and fiber properties in the Çukurova region, Turkey. Field Crops Research, 78: 141-149.

Grespan, S.L.; Zancanaro, L.

1999 Nutrição e Adubação do Algodoeiro no Mato Grosso. In: Fundação-MT. Mato Grosso: Liderança e Competitividade. Rondonópolis: Fundação MT; Campina Grande: EMBRAPACNPA, 182 p. (Fundação MT. Boletim, 3).

Lauchli, A; Kent, L.M.; Turner, J.C.

1981 Physiological responses of cotton genotypes to salinity. Proc. Beltwide Cotton Prod. Res. Conf. p. 40.

Maas, E.V.; Hoffman, G.J.

1977 Crop salt tolerance-current assessment. Journal of the irrigation and Drainage Engineering, 103: 115-134.

Malavolta, E.; Vitti, G.C.; Oliveira, S.A.

1997 Avaliação do estado nutricional das plantas: princípios e aplicações. 2.ed. rev. atual. Piracicaba: POTAFÓS, p. 319.

Mateo, M.T.

1999 Efecto de Distintos Niveles de Salinidad en el Agua de Riego sobre el Desarrollo y Contenido Iónico de Plantas de Phoenix canariensis. Thesis, Universidad de Murcia. p. 105.

Munns, R.; Tester, M.

2008 Mechanisms of salinity tolerance. Annual Review of Plant Biology, 59: 651-681.

Pettigrew, W.T.; Meredith Jr., W.R.

1997 Dry matter production, nutrient uptake, and growth of cotton as affected by potassium fertilization. Journal of Plant Nutrition, 20: 531-548.

Raij, B.Van; Cantarella, H.; Guaggio, J.A.; Furlani, A.M.C.

1996 Recomendações de adubação e calagem para o estado de São Paulo, 2ed. Campinas, Instituto Agronômico e Fundação IAC. p. 285 (Boletim Técnico 100).

Read, J.K.; Reddy, R.; Jenkins, J.N.

2006 Yield and fiber quality of Upland cotton as influenced by nitrogen and potassium nutrition. European Journal of Agronomy, 24: 282-290.

Reddy, K.R.; Zhao, D.

2005 Interactive effects of elevated $\mathrm{CO}_{2}$ and potassium deficiency on photosynthesis, growth and biomass partitioning of cotton. Field Crops Res., 94: 201-213.

Rosolem, C.A.

2001 Problemas em nutrição mineral, calagem e adubação do algodoeiro. Botucatu: Unesp, 16 p. (Informações Agronômicas, no 95.).

Sabino, N.P.; Kondo J.I.; Silva, N.M.; Sabino, J.C.; Igue, T. 1995 Efeitos da calagem e da adubação potássica sobre características agronômicas e propriedades tecnológicas da fibra do algodoeiro. Bragantia, 54: 385-392.

Shannon, M.C.; Grieve, C.M.

1998 Tolerance of vegetable crops to salinity. Scientia Horticulturae, 78: 5-38.

Silva, N.M.

2001 Calagem e adubação do algodoeiro. In: III Congresso Brasileiro de Algodão, Resumo das palestras. Dourados (MS): UFMS e Embrapa Agropecuária Oeste, p. 155-157.

Ting, I.P 1982 Plant Physiology. London: Addison-Wesley, 642 p. 